\title{
Analysis of GCR Spectra and Composition Using Penetrating Particle Data from the CRIS Instrument on ACE
}

\author{
M. E. Wiedenbeck ${ }^{*} a$, W. R. Binns ${ }^{b}$, E. R. Christian ${ }^{c}$, A. C. Cummings ${ }^{d}$, A. J. Davis ${ }^{d}$, \\ G. A. de Nolfo ${ }^{c}$, M. H. Israel ${ }^{b}$, A. W. Labrador ${ }^{d}$, R. A. Leske ${ }^{d}$, R. A. Mewaldt ${ }^{d}$, \\ E. C. Stone ${ }^{d}$, and T. T. von Rosenvinge ${ }^{c}$ \\ a Jet Propulsion Laboratory, California Institute of Technology \\ Pasadena, CA 91109 USA \\ ${ }^{b}$ Washington University \\ St. Louis, MO 63130 USA \\ ${ }^{c}$ NASA / Goddard Space Flight Center \\ Greenbelt, MD 20771 USA \\ ${ }^{d}$ California Institute of Technology \\ Pasadena, CA 91125 USA \\ E-mail: mark.e.wiedenbeck@jpl.nasa.gov
}

\begin{abstract}
The Cosmic Ray Isotope Spectrometer (CRIS) on NASA's Advanced Composition Explorer (ACE) spacecraft has been making precise measurements of cosmic-ray elemental and isotopic composition and energy spectra for nearly 18 years. This instrument uses the $d E / d x$ versus total energy technique to identify nuclei that stop in thick stacks of silicon solid-state detectors and to measure their energy. The energy range covered for these stopping particles extends up to $\sim 280$ $\mathrm{MeV} /$ nuc for $\mathrm{O}$ and $\sim 570 \mathrm{MeV} /$ nuc for Fe. We have developed a new technique for identifying particles that penetrate the entire detector stack that relies on a combination of the total energy deposited in the stack and the change of $d E / d x$ from the front to the back of the stack. This technique allows us to extend energy spectra for cosmic-ray elements to higher energies and can be used for bridging the energy gap between the CRIS stopping-particle spectra and measurements made in low-Earth orbit by instruments such as HEAO-C2, PAMELA, and AMS-02. We describe the technique for assigning atomic number and energy to penetrating particle events and discuss the corrections needed for deriving energy spectra from these data.
\end{abstract}

The 34th International Cosmic Ray Conference,

30 July- 6 August, 2015

The Hague, The Netherlands

\footnotetext{
* Speaker.

$\dagger$ The JPL author's copyright for this publication is held by the California Institute of Technology. Government Sponsorship acknowledged.
} 


\section{Introduction}

The Cosmic Ray Isotope Spectrometer (CRIS) instrument on the Advanced Composition Explorer (ACE) spacecraft has been returning detailed measurements of galactic cosmic ray (GCR) energy spectra and composition for elements from $\mathrm{B}$ (atomic number $Z=5)$ through $\mathrm{Ni}(Z=28)$ between $\sim 50$ and $\sim 500 \mathrm{MeV} /$ nuc since August $1997^{1}$. The measurements are made using the $d E / d x$ versus total-energy technique in a sensor system consisting of stacks of silicon solid-state detectors in combination with a scintillating optical fiber trajectory (SOFT) sensor that determines incidence directions for the particles. The instrument is described in detail in [1] and a simplified cross section is shown in the left-hand panel of Figure 1.
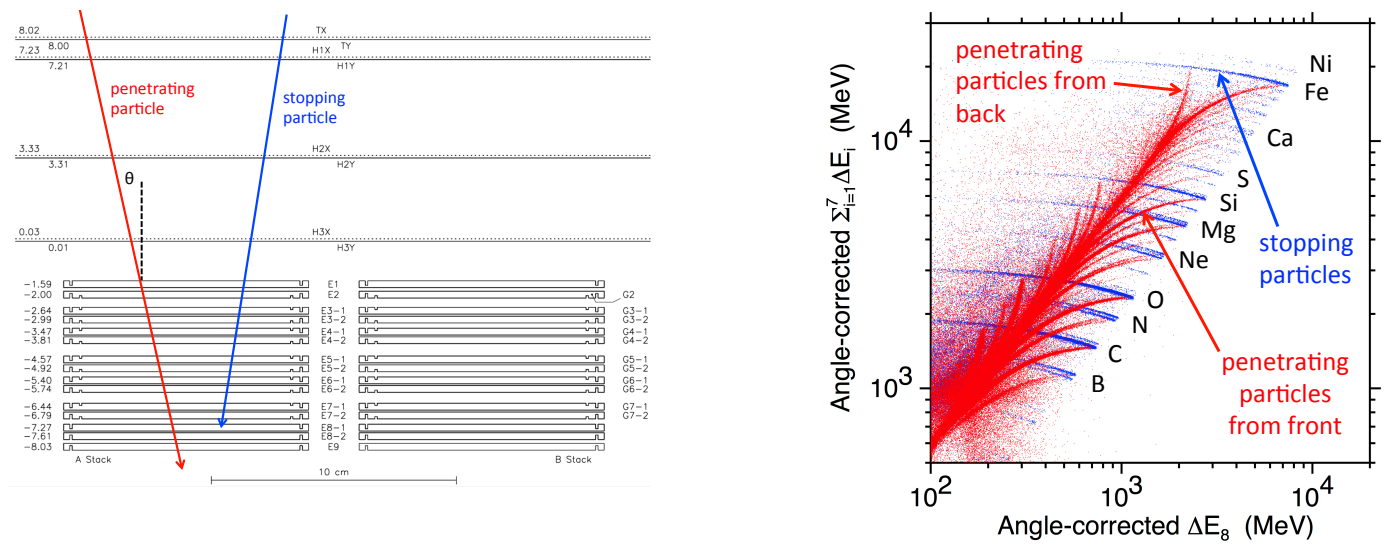

Figure 1: Left: Cross-sectional view of the ACE/CRIS instrument showing two of the four silicon detector stacks below the scintillating optical fiber (SOFT) hodoscope. Example trajectories for a particle stopping in the E8 detector and a particle that penetrates the entire telescope are illustrated. Right: Scatter plot of two months of CRIS data from 2009 including particles that triggered all detectors down through E8 and either did not trigger E9 (stopping particles, shown in blue) or did trigger E9 (penetrating particles, overplotted in red). For clarity only $10 \%$ of the penetrating events are included. A simple multiplicative correction factor that depends only on the measured incidence angle has been applied to approximately correct for the angular dependence of the particle tracks. Data cuts have been made to select good trajectories but no consistency cuts among silicon detector signals have been applied. Tracks due to particles that penetrated the stack from back to front can also be seen.

In the normal application of the $d E / d x-E$ technique, we analyze particles that pass through SOFT, enter the silicon detector stack through the E1 detector, and stop somewhere in the stack between E2 and E8, inclusive. For such particles the total energy $E$ at the top of the silicon stack is derived by adding the energy losses in the silicon detectors. The bottom detector in the stack, E9, is used to identify particles that do not satisfy the requirement for stopping in the stack. These "penetrating particle" events are also recorded, but are not treated in our standard analysis. The right-hand panel of Figure 1 shows a plot of energy loss $(\Delta E)$ measured using E1 through E7 versus residual energy $\left(E^{\prime}\right)$ measured in $\mathrm{E}$, with a simple correction for angle of incidence applied to both $\Delta E$ and $E^{\prime}$ (see Section 3.2 of [2]). On the $\Delta E-E^{\prime}$ plot, events for which E9 was not

\footnotetext{
${ }^{1}$ Data available from http://www.srl.caltech.edu/ACE/ASC/level2/lvl2DATA_CRIS.html
} 
triggered are shown in blue. Those for which E9 was triggered are overplotted in red. Thus our standard analysis addresses only the blue portions of these tracks.

At energies not too much greater than required to penetrate the stack the tracks for different elements are still distinct, but at higher energies they merge and the elements can no longer be distinguished. At sufficiently high energies a nucleus penetrating the stack will lose only a small fraction of its energy and its specific ionization, $d E / d x$, will not change appreciably from the front to the back of the stack. In this case the $\Delta E$ and $E^{\prime}$ signals are just proportional to one another, yielding a point that falls along the diagonal. In first approximation the $d E / d x$ of a heavy nucleus is proportional to $Z^{2} / v^{2}$, where $Z$ is its atomic number and $v$ is its velocity. Thus in the high-energy region there can be ambiguities in distinguishing fast (high energy per nucleon), high- $Z$ particles from slower (lower energy per nucleon), lower- $Z$ particles.

When the slowing of a particle from the front to the back of the detector stack results in a measurable increase in its $d E / d x$, one can attempt to use the shape of the curve of $d E / d x$ versus depth in the stack to derive values of both $Z$ and $E / M$. Using ACE/CRIS penetrating particle data for several of the most abundant elements, Labrador et al. [3] selected regions of a $\Delta E-E^{\prime}$ plot where the tracks for those elements are well resolved from the tracks for other elements and used a range-energy relation to model the pattern of energy losses in E1 through E8 as a function of $E / M$ given the $Z$ value for the element being considered. A comparison between the measured pattern of energy losses in E1 through E8 with the model calculations then allowed the derivation of the best-fit $E / M$ value for each event.

In this paper we address the problem of extending the penetrating particle analysis to include rare as well as abundant elements. Our approach is to use a different set of measured quantities to make a two-dimensional scatter plot in which the tracks of penetrating heavy nuclei are better separated than on the conventional $\Delta E$ versus $E^{\prime}$ plot used for stopping particles. Once the particle has been identified, then its energy per nucleon can easily be calculated given the total energy deposited in the silicon stack, the thickness of the stack, and the measured angle of incidence. Backgrounds can be rejected by comparing the pattern of energies deposited in individual detectors with the pattern expected from the $Z$ and $E / M$ values that have been assigned.

\section{Motivation for Parameter Selection}

To provide some insight into a parameterization that could result in well resolved penetratingparticle tracks, we start by using a power-law approximation to the heavy-ion range-energy relation, $R=\left(k M / Z^{2}\right)(E / M)^{a}$, where $Z, M, E$, and $R$ are the particle's atomic number, mass number, energy, and range in silicon. The parameters $a$ and $k$ specify the power law and have approximate values $a \simeq 1.6$ and $k \simeq 0.0070$ when $E$ is expressed in $\mathrm{MeV}$ and $R$ in $\mathrm{g} / \mathrm{cm}^{2}$ of silicon. From the range-energy power law one obtains an expression for the specific ionization, $S$, as a function of $Z$, $M$, and $R$ :

$$
S_{Z, M}(R)=\frac{1}{a}\left(\frac{Z^{2} M^{a-1}}{k}\right)^{1 / a} R^{1 / a-1} .
$$

For a detector stack with a total thickness $L\left(\mathrm{~g} / \mathrm{cm}^{2}\right.$ of silicon) and a particle penetrating the stack at an angle $\theta$ relative to the detector normal, the fractional change in the specific ionization 
going from the front to the back of the stack can be expressed in the power-law approximation as

$$
\frac{\Delta(d E / d x)}{(d E / d x)_{0}} \equiv \frac{S_{Z, M}(R-L \sec \theta)-S_{Z, M}(R)}{S_{Z, M}(R)}=\left(1-\frac{L \sec \theta}{R}\right)^{1 / a-1}-1 .
$$

Thus in this approximation the fractional change in $d E / d x$ is just a measure of the fraction of the particle's range that is covered by the detector stack (including the $\sec \theta$ dependence) and is independent of the particle species.

As a second parameter we use $\left(\Delta E_{\text {total }} / \sec \theta\right)^{1 / 2}$ where $\Delta E_{\text {total }}$ is the total energy deposited in the detector stack (E1 through E8). Note that $\Delta E_{\text {total }} / L \sec \theta$ is simply the mean value of $d E / d x$ in the detector stack. Calculating $\left(\Delta E_{\text {total }} / \sec \theta\right)^{1 / 2}$ in the power-law approximation we have

$$
\left(\frac{\Delta E_{\text {total }}}{\sec \theta}\right)^{1 / 2}=\left\{\left(\frac{Z^{2}}{k M}\right)^{1 / a} \frac{M}{\sec \theta}\left[R^{1 / a}-(R-L \sec \theta)^{1 / a}\right]\right\}^{1 / 2} .
$$

For fixed values of $R$ and $\theta$ (and thus a fixed value of $\Delta(d E / d x) /(d E / d x)_{0}$ defined in Eq. 2.2), $(d E / d x)^{1 / 2}$ is proportional to $Z^{1 / a} M^{(1-1 / a) / 2}=Z^{0.625} M^{0.187} \simeq Z^{0.812}$, where the final approximate equality assumes $M \propto Z$.

The expected shapes of the penetrating particle tracks on a plot of $\Delta(d E / d x) /(d E / d x)_{0}$ versus $\left(\Delta E_{\text {total }} / \sec \theta\right)^{1 / 2}$ are shown in the left panel of Figure 2 for three different angles of incidence, $0^{\circ}, 30^{\circ}$, and $45^{\circ}$, where a stack thickness $L=9.79 \mathrm{~g} / \mathrm{cm}^{2}$ ( $42 \mathrm{~mm}$ of silicon) is assumed. For all but the lightest elements, the track spread caused by the angle dependence would significantly degrade the element separation if data covering this broad range of angles were combined. We have derived a simple, angle-dependent correction to the track locations that reduces this spread. In the powerlaw approximation $D$, the partial derivative of $\left(\Delta E_{\text {total }} / \sec \theta\right)^{1 / 2}$ with respect to $\sec \theta$ for constant $\Delta(d E / d x) /(d E / d x)_{0}$, can readily be calculated (keeping in mind the fact that $\Delta E_{\text {total }}$ depends on $\sec \theta)$. Multiplying each abscissa value by $1-D(\sec \theta-1)$ will shift the point's horizontal position so that it approximately coincides with the point on the $0^{\circ}$ track that has the same value of $\Delta(d E / d x) /(d E / d x)_{0}$. As shown in the right panel of Figure 2, the tracks for all angles between $0^{\circ}$ and $45^{\circ}$ collapse onto a common track narrow enough to allow separation of adjacent elements (remembering that tracks are plotted in Figure 2 only for even- $Z$ elements).

\section{Application to the ACE/CRIS Penetrating Particle Data}

In order to apply this technique to data from the ACE/CRIS instrument we replace the parameters being plotted with closely related quantities that are readily derived from the measurements. For the ordinate we replace $\Delta(d E / d x) /(d E / d x)_{0}$ by $\left[(d E / d x)_{E 8}-(d E / d x)_{E 1}\right] /(d E / d x)_{E 1}$ where $(d E / d x)_{E 1}$ is the energy loss in the E1 detector divided by the particle pathlength (including $\sec \theta$ factor) in the active thickness of that detector and similarly for $(d E / d x)_{E 8}$. For the abscissa we use $\left[\sum_{i=1}^{8}(\Delta E)_{E i} / \sec \theta\right]^{1 / 2}$ where $(\Delta E)_{E i}$ is the measured energy loss in detector $E i$ with a small correction to account for the energy deposited in the detector's dead layers. Figure 3 shows CRIS data from 2009 for three ranges of $\theta$. In the right-hand panel we have included the angle correction that was derived based on a power-law range-energy relation. The exponent in that relation was adjusted to give the best overlap of the tracks for the three different angle ranges that are shown. 


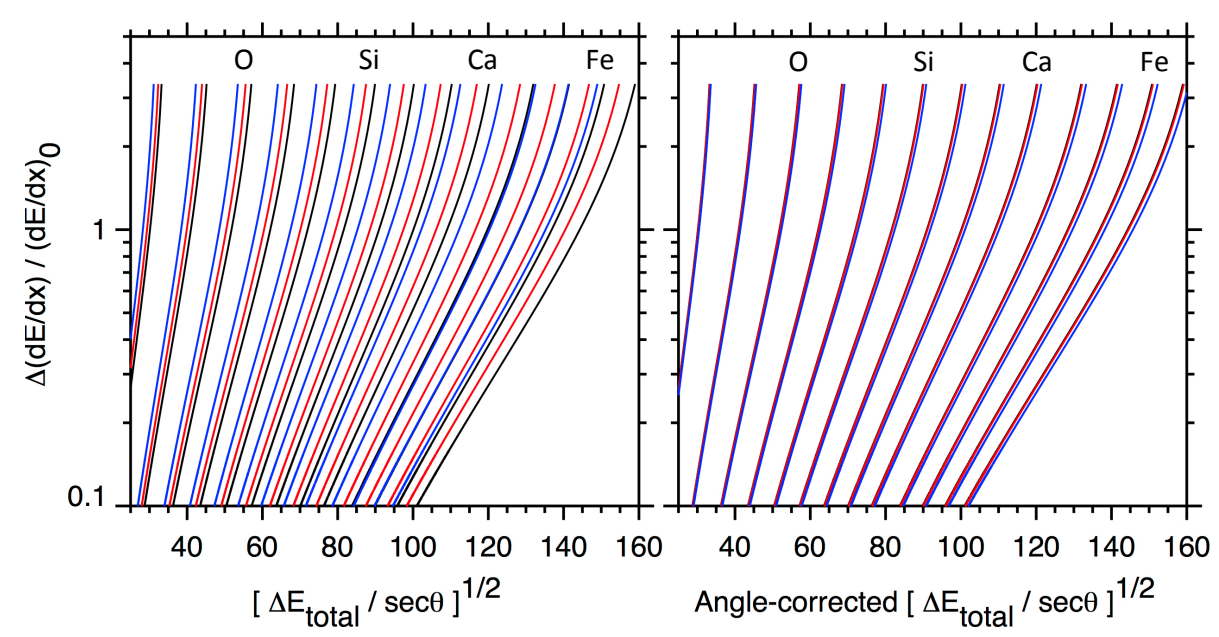

Figure 2: Illustration in the power-law approximation of two-parameter plots used for obtaining separated element tracks for penetrating particles. The two panels are identical except that a correction for particle angle of incidence has been applied in the right-hand panel. Tracks are shown for even $Z$ elements from Be $(Z=4)$ through $\mathrm{Ni}(Z=28)$. Track colors indicate the angles of incidence: black, $0^{\circ}$; red, $30^{\circ}$; blue, $45^{\circ}$. On the right, the red and blue tracks nearly overlap the black tracks.

Comparing the plots in Figures 2 and 3 one sees clear differences in the shapes and spacings of the tracks. This reflects the inadequacy in the power-law approximation used for calculating the tracks in Figures 2. The exponent that we used, $a=1.6$, is approximately correct for energies around 300-400 MeV/nuc, but varies by $\sim 0.35$ between $100 \mathrm{MeV} /$ nuc and $1000 \mathrm{MeV} /$ nuc.
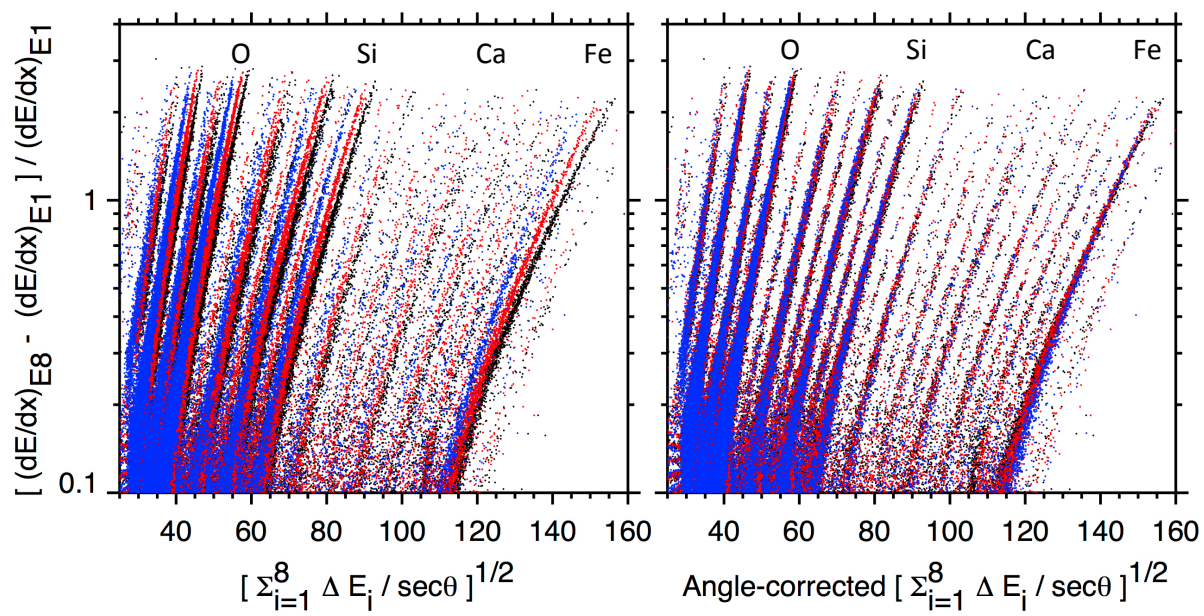

Figure 3: ACE/CRIS penetrating particle data for incidence angle ranges of $0^{\circ} \leq \theta<7^{\circ}$ (black), $29^{\circ} \leq \theta<$ $31^{\circ}$ (red), and $43^{\circ} \leq \theta<47^{\circ}$ (blue). The left panel contains no correction for incidence angle while the right panel includes this correction. Cuts have been applied to select good trajectories. The only cut made on the silicon detector pulse heights was a requirement that the smallest measured single-detector $d E / d x$ value in an event not be less than $0.6 \times$ the average of all of the $d E / d x$ measurements in that event. 


\section{Derivation of Incident Energy per Nucleon}

On a plot of the type shown on the right in Figure 3, one can select regions containing an individual element. For values of the ordinate below $\sim 0.2$ (i.e., for an increase in $d E / d x$ of less than $\sim 20 \%$ from the front to the back of the stack) overlap between elements becomes a possibility. In this high-energy region an analysis that goes beyond that presented in this paper will be needed. An expanded version of the angle-corrected plot is shown in the left-hand panel of Figure 4 with a polygonal region used to select the oxygen track. Knowing that $Z=8$ and assuming that $M=16$ for all of the particles in this region one can then calculate the energy per nucleon at the front of the silicon detector stack using

$$
\Delta E_{\text {total }}=M\left[\mathscr{E}_{Z, M}\left(L \sec \theta+R^{\prime}\right)-\mathscr{E}_{Z, M}\left(R^{\prime}\right)\right]
$$

Here $\mathscr{E}_{Z, M}(R)$ is a function giving the energy per nucleon for a nucleus of charge $Z$, mass $M$, and range $R$ in silicon. The quantity $R^{\prime}$, which represents the range of the particle beyond the back of the silicon stack, is the only unknown in this equation. It can easily be derived by means of a binary search starting with trial values for $R^{\prime}$ of 0 and $R_{\max }^{\prime}$. Since, as mentioned above, the $\Delta(d E / d x) /(d E / d x)_{0}$ depends mainly on $R / L \sec \theta$, the minimum value of the ordinate can be used to estimate a suitable value for $R_{\max }^{\prime}$. In our example we used $R_{\max }^{\prime}=4 \times(L \sec \theta)_{\max }$.

The right-hand panel of Figure 4 shows the same data as the left-hand panel, but now plotted as a function of $E / M$, the derived energy per nucleon at the front of the silicon detector stack. As expected, the energy interval is shifted to higher energies for wider-angle particles.

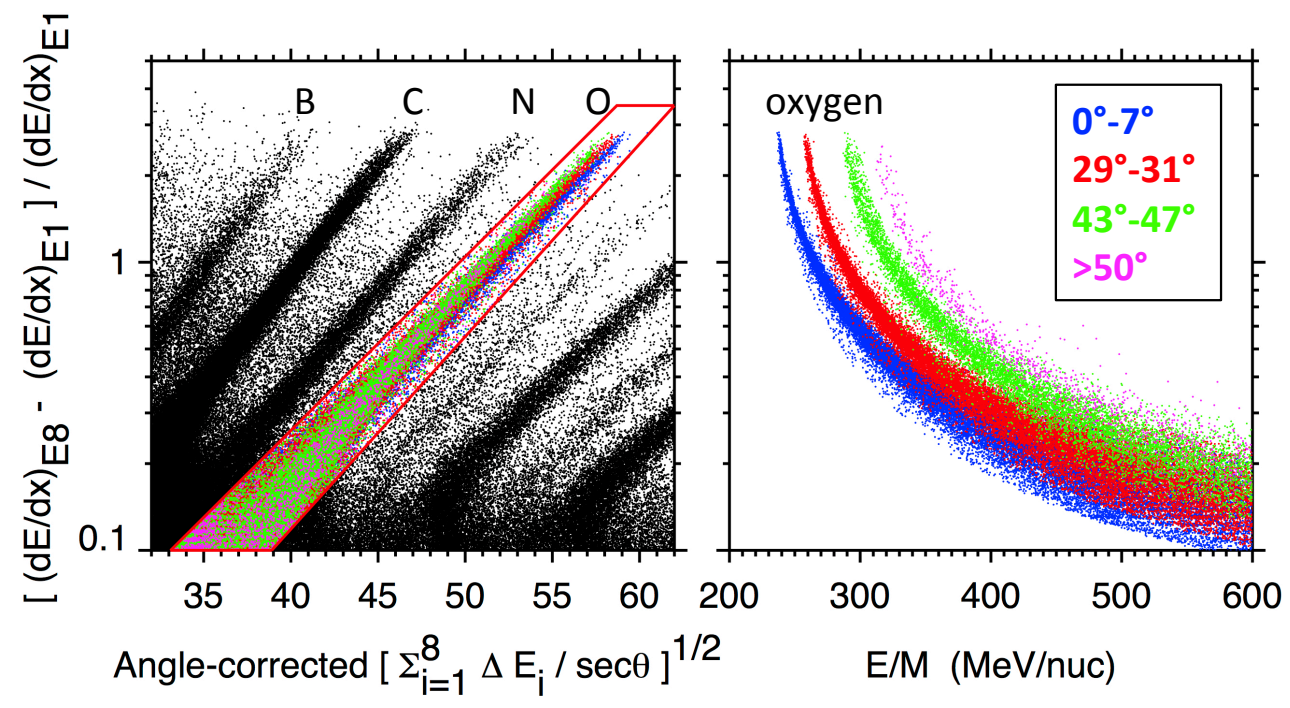

Figure 4: Left: Expanded version of the region of the plot near the oxygen track. Particles inside the polygon are analyzed as ${ }^{16} \mathrm{O}$. Particles are shown for four intervals of incidence angle, as indicated by colors identified in the right-hand panel. Right: Oxygen data replotted as a function of the values derived for $E / M$ at the front of the silicon stack. 


\section{Remaining Steps}

Using the values of $Z, M$ (assumed based on $Z$ ), $\sec \theta, E / M$, and the thicknesses of all of the silicon layers (both active and dead) that were penetrated, the expected energy losses in each active layer can be calculated. A $\chi^{2}$ can be constructed comparing the measured and calculated energy deposits and taking into account the sources of error, which should be dominated by Bohr/Landau fluctuations in most cases. A $\chi^{2}$ test can be used to reject bad events such as may occur when an incident particle suffers a nuclear collision in the stack and undergoes fragmentation. Some investigation will be needed of the extent to which high-energy knock-on electrons passing from one layer to the next can alter the calculated pattern of energy losses.

Having derived values of $Z$ and $E / M$ for individual penetrating particles and applied cuts to eliminate background events, there remain several additional steps that must be taken in order to obtain high-energy extensions of the CRIS elemental energy spectra based on the penetrating particle data. These are very similar to the steps normally used in the stopping particle analysis and described in [5, 4], and can be summarized as follows:

1. energies derived for particles entering the silicon stack are corrected to the top of the SOFT sensor to obtain the incident particle energies,

2. count rates accumulated on board are used to correct for readout inefficiencies,

3. the measured live time is used to convert from counts to event rates,

4. a correction is applied for the SOFT sensor's detection efficiency, which is close to $100 \%$ for particles having $d E / d x \times \sec \theta \gtrsim 100 \mathrm{MeV} /\left(\mathrm{g} / \mathrm{cm}^{2}\right)$ [4] but decreases for less heavily ionizing particles,

5. calculated corrections are applied to account for particles lost due nuclear interactions in the instrument,

6. the measured event rates are divided by the calculated instrument geometrical factor for penetrating particles (taking into account any data cuts made based on the trajectories derived from SOFT) to obtain particle intensities,

7. a correction is applied to the element spectra to take into account the actual isotopic composition of the element, which is derived from the stopping-particle data.

For each element there will be an interval of $E / M$ in which wide-angle particles stop and narrow-angle particles penetrate. Using the energy dependences of the stopping- and the penetratingparticle geometrical factors it is possible to obtain intensity measurements in this overlap region from both data sets, thus allowing cross checks between the two analyses. Ultimately the two data sets can be combined to derive energy spectra extending from the lowest energies detected for stopping particles up to the point at high energy where penetrating-particle element tracks begin to overlap. Figure 5 shows the upper and lower energy limits for the analysis of stopping particles with atomic number $4 \leq Z \leq 28$ incident at angles of $0^{\circ}$ and $45^{\circ}$. Also shown is the extension of the CRIS upper energy limits that is possible using the penetrating particle data, assuming that element identification is possible when the increase of $d E / d x$ from the front to the back of the detector stack is at least $10 \%$. As suggested by Figure 3, for some rare elements it may be necessary to restrict the penetrating particle analysis to energies for which $d E / d x$ increases by at least $\sim 20 \%$. 


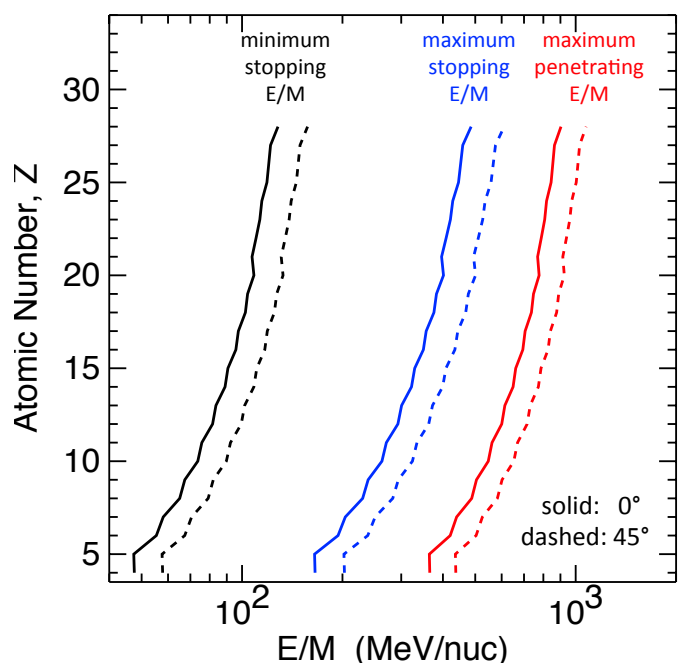

Figure 5: Energy intervals covered by the CRIS instrument for stopping particles (black: lower limit; blue: upper limit) and extension of the upper energy limit (red) by using penetrating particles for which $d E / d x$ changes by at least $10 \%$ from the front to the back of the silicon detector stack. Solid (dashed) curves show limits for particles incident at $0^{\circ}\left(45^{\circ}\right)$ from the normal to the detector surfaces.

By taking advantage of the large set of CRIS penetrating-particle event data accumulated since the start of the ACE mission, we expect to be able to extend our measurements of GCR element spectra by several hundred $\mathrm{MeV} /$ nuc. In addition to facilitating comparisons with data from higherenergy cosmic-ray experiments, these spectra will allow more-detailed studies of solar modulation effects extending over nearly two full solar cycles.

\section{Acknowledgments}

We thank Nathan Yanasak, Jeff George, and Kelly Lave for their extensive work on obtaining elemental spectra from the CRIS stopping particle data and in developing the corrections needed for deriving cosmic-ray intensities. This work was supported by NASA at JPL, Caltech, Washington Univ., and NASA/GSFC.

\section{References}

[1] E. C. Stone et al., The Cosmic-Ray Isotope Spectrometer for the Advanced Composition Explorer, Space Sci. Rev., 86, 285, 1998.

[2] R. A. Mewaldt et al., The Low-Energy Telescope (LET) and SEP Central Electronics for the STEREO Mission, Space Sci. Rev., 136, 285, 2008.

[3] A. W. Labrador et al., Extended Energy Spectrum Measurements of Elements with the Cosmic Ray Isotope Spectrometer (CRIS), Proc. 28th Internat. Cosmic Ray Conf. (Tsukuba), 4, 1773, 2003.

[4] K. A. Lave et al., Galactic Cosmic-ray Energy Spectra and Composition during the 2009-2010 Solar Minimum Period, ApJ, 770, 117, 2013.

[5] J. S. George et al., Elemental Composition and Energy Spectra of Galactic Cosmic Rays during Solar Cycle 23, ApJ, 698, 1666, 2009. 